\title{
EFECTO DEL APAGADO DE LA RESISTENCIA DE LA NACEDORA SOBRE LA CALIDAD DEL POLLITO
}

\author{
Frank Steven Ávila-Oviedo, Est. MVZ, Javier Enrique Vargas-Bayona, MVZ, \\ Javier Eduardo Nieto-Pico ${ }^{\star}, \mathrm{MVZ}_{1}$, Esp. (c)
}

${ }_{1}$ Facultad de Medicina Veterinaria y Zootecnia, Universidad Cooperativa de Colombia, sede Bucaramanga, Colombia

Recibido: 6 de febrero del 2013 Aprobado: 24 de abril del 2013

* Autor de correspondencia: Javier Eduardo Nieto Pico, Facultad de Medicina Veterinaria y Zootecnia, Universidad Cooperativa de Colombia, Bucaramanga, Colombia, calle 30A n.3 33-51, of. 603, (57) 7 6356624, correo electrónico: javier.nieto@ucc.edu.co

Cómo citar este artículo: Ávila-Oviedo FS, Vargas-Bayona JE, Nieto-Pico JE. Efecto del apagado de la resistencia de la nacedora sobre la calidad del pollito. Spei Domus. 2013; 9(18): 9-14.

Resumen. El estudio pretendió evaluar la calidad del pollito cuando se modificó la temperatura de la máquina nacedora al final del ciclo de incubación. El experimento se realizó en una sola máquina nacedora (chick master ${ }^{\circledast}$, génesis III) con capacidad para nacimiento de 15.552 pollos. Se realizaron tres ensayos: primero se apagó la resistencia de la máquina nacedora 48 horas antes del nacimiento; luego, 24 horas antes, y en el tercero no se apagó la resistencia de la máquina. Se utilizó el mismo lote de huevos de reproductoras Cobb-Avian 48 con edades de 32 y 33 semanas. Se evaluó la cicatrización del ombligo, pollito de primera (\%), pollito de segunda (\%), descarte (\%), deshidratación (\%). Para la realización del embrio-diagnóstico se tuvo en cuenta la mortalidad tardía de pollitos y picados no nacidos. El análisis estadístico fue descriptivo para cada una de las variables y se apoyó con el examen test físico de Cervantes en los pollitos nacidos. En el primer ensayo la cicatrización de ombligos fue de $93,8 \%$ en aves de primera, $0,5 \%$ de segunda y $0,5 \%$ de descarte; en el segundo $94,2 \%$ en aves de primera, $0,4 \%$ de segunda y $0,7 \%$ de descarte; en el tercero $92,9 \%$ en aves de primera, $0,7 \%$ de segunda y $0,6 \%$ de descarte. Se observó que la resistencia encendida todo el tiempo causó deshidratación en el $8 \%$ de los animales y un aumento de $0,2 \%$ en la mortalidad tardía y de $0,6 \%$ en malas posiciones con signos de estrés calórico.

Palabras clave: deshidratación, embrio-diagnóstico, ombligos, porcentaje nacimiento, temperatura.

\section{Effect of Cutting off Power to the Hatcher on the Quality of Chicks}

\begin{abstract}
The study aimed to evaluate chick quality when the temperature was changed at the end of the incubation cycle. The experiment was performed on a single machine hatcher (chick master ${ }^{\circledR}$, genesis III), with a capacity for births of as many as 15,552 chickens. There were 3 trials: in the first one, power was turned off to the hatcher machine 48 hours before birth; for the second trial it was turned off 24 hours prior to birth; finally, for the third trial power was not turned off. The same batch of Cobb-Avian 48 egg layers ages 32 and 33 weeks was used. The percentage of navel healing was assessed for the first chicks, second chicks along with the discard percentage and dehydration percentage. In carrying out embryo diagnosis, late mortality and unborn chicks were taken into account. Statistical analysis was descriptive for each variable and was based on the Cervantes physical examination test performed on the hatched chicks. In the first trial, navel healing was $93.8 \%$ in first chicks, $0.5 \%$ in second chicks and $0.5 \%$ among discards. In the second trial, navel healing was $94.2 \%$ in first chicks, $0.4 \%$ in second chicks and $0.7 \%$ among discards. In the third trial, the figures were $92.9 \%$ among first chicks, $0.7 \%$ among second chicks and $0.6 \%$ among discards. It was found that when the power was always on, there was dehydration among $8 \%$ of the animals and a $0.2 \%$ increase in late mortality along with $0.6 \%$ in bad positions with signs of heat stress.
\end{abstract}

Keywords: dehydration, embryo-diagnosis, navels, birth rate, temperature.

\section{Efeito do desligamento da resistência da incubadora sobre a qualidade do pintinho}

Resumo. Este estudo pretendeu avaliar a qualidade do pintinho quando se modificou a temperatura ao final do ciclo de incubação. O experimento se realizou em uma só máquina incubadora (chick master ${ }^{\circledR}$, gênese III) com capacidade para nascimento de 15.552 frangos. Realizaram-se três ensaios: primeiro se desligou a resistência da máquina nascedora 48 horas antes do nascimento; no segundo, 24 horas antes e, no terceiro, não se desligou a resistência da máquina. Utilizou-se o mesmo lote de ovos de reprodutoras Cobb-Avian 48 com idade de 32 e 33 semanas. Avaliou-se a cicatrização do umbigo, pintinho de primeira (\%), pintinho de segunda (\%), descarte (\%), desidratação (\%). Para a realização do embriodiagnóstico, considerou-se a mortalidade tardia de pintinhos e quebrado não nascidos. A análise descritiva foi uma das variáveis e apoiou-se com o exame teste físico de Cervantes nos pintinhos nascidos. No primeiro ensaio, a cicatrização de umbigos foi de $93,8 \%$ em aves de primeira; $0,5 \%$ de segunda e $0,5 \%$ de descarte. No segundo $94,2 \%$ em aves de primeira; $0,4 \%$ de segunda e $0,7 \%$ descarte. No terceiro $92,9 \%$ em aves de primeira; $0,7 \%$ de segunda e $0,6 \%$ em descarte. Observou-se que a resistência ligada todo o tempo causou desidratação em $8 \%$ dos animais e um aumento de $0,2 \%$ na mortalidade tardia e de $0,6 \%$ em más posições com signos de estresse calórico.

Palavras-chave: desidratação, embriodiagnóstico, umbigos, porcentagem nascimento, temperatura. 


\section{Introducción}

La producción avícola mundial se ha incrementado a una tasa constante y relativamente rápida desde los sesenta, siguiendo diferentes ritmos según las características propias del desempeño de la economía de cada país [1]. En Colombia, las empresas avícolas le apostaron al crecimiento, expansión y la productividad, convirtiendo al gremio en el de mayor crecimiento en el sector agropecuario, como lo demuestra Fenavi en su estudio económico, al indicar que en Colombia entre el 2005 y el 2010 la industria creció en un 28,5\% [2].

El sector avícola es una cadena productiva completa y compleja en el que el desempeño y la eficiencia de cada eslabón condicionan el rendimiento del otro. De esta manera, un pollo tipo boiler de rendimiento óptimo en la granja de engorde depende de un buen proceso de incubación, y este a su vez dependerá de la calidad del huevo proveniente de la granja de reproductoras [3].

La producción de pollitos de alta calidad es un proceso complejo que involucra desde el manejo de las reproductoras en aspectos como nutrición, nivel de anticuerpos contra enfermedades prevalentes, prácticas de manejo y conservación del huevo, hasta el proceso en la incubadora y posterior transporte del pollito para que finalmente sea entregado en la granja, donde seguirá su proceso [4].

El máximo rendimiento productivo que puede ser obtenido de un pollo al final del ciclo de engorde depende en gran medida de la calidad del pollito que sale de la planta de incubación, debido a que su manejo allí determina la capacidad para mostrar y desarrollar todo su potencial [4]. Algunas investigaciones han establecido la evidente relación que existe entre la incubación, la crianza del pollito en sus primeros días de vida y la posibilidad de obtener el máximo rendimiento productivo [5].

La incubación está influenciada por diversos factores como producción de calor del embrión, tiempo de depósito del huevo después de la postura cuyo periodo puede ser de dos a tres días, edad de las reproductoras, época del año (temporada húmeda o seca), flujo de aire a través de la masa de huevo, incubación en sistema de carga única o múltiple y la línea genética [6]; pero el factor físico más importante es la temperatura, que condiciona los otros factores como ventilación y humedad, y va a determinar el porcentaje de nacimientos y la calidad del pollito [5].
La exposición a temperaturas adversas (estrés por frío o por calor) influye negativamente en el desempeño de los pollitos. En cuanto a la manipulación térmica durante la vida embrionaria o en los primeros días post-eclosión, la temperatura actúa sobre el sistema de control termorregulador del ave, aumentando o eliminando la termotolerancia por modulación de producción de calor [7].

Expertos en genética hacen una selección según el índice de crecimiento, en el que aumenta el crecimiento embrionario y la producción de calor metabólico, lo que hace más difícil conseguir una incubación óptima. La mayor producción de calor metabólico de los embriones requiere una mayor capacidad de enfriamiento, por lo que supondrá un área de interés primordial para las mejoras en las tecnologías y prácticas de incubación. En la actualidad, existen muy pocas incubadoras que disponen de una capacidad de enfriamiento suficiente como para optimizar el proceso de incubación en función del progreso genético [8]. Por ello se hace necesario buscar alternativas que eviten el estrés térmico de los embriones.

La experiencia práctica y la investigación científica demuestran que el control de la temperatura embrionaria permite mejor incubabilidad y calidad de pollito. Especialmente influirá en la utilización de la yema y en la cicatrización del ombligo, produciendo diferencias en la mortalidad que se presenta en la primera semana de vida, debido a las infecciones del ombligo-vitelo $y$ otras infecciones [9].

Para contrastar la literatura científica y la experiencia práctica respecto a que la disminución de la temperatura de la máquina nacedora al final del periodo de incubación permite obtener pollitos de mejor calidad, se apagó la resistencia, permitiendo que el calor embrionario mantuviera la temperatura dentro de la máquina nacedora. Los pollitos de un día son un punto crítico entre las granjas de reproductoras y las granjas de engorde [10]; por tanto, mejorar el proceso de incubación tiene como finalidad obtener pollos viables al menor costo de producción posible [11].

El desarrollo de este proyecto de investigación tuvo como objetivo evaluar el efecto del apagado de la resistencia de la máquina nacedora 24 y 48 horas antes del nacimiento del pollito, evaluando diferentes variables de calidad como cicatrización del ombligo, mortalidad, deshidratación, calidad del pollito, entre otras. Con esto se busca ofrecer una alternativa de mejoramiento de la calidad del pollito recién nacido para las 
empresas incubadoras, que en muchas ocasiones ven disminuida la calidad de las aves recién nacidas debido al estrés calórico que se genera en la máquina incubadora al momento del nacimiento, lo que, como fue mencionado anteriormente, condiciona la capacidad productiva de un animal.

\section{Materiales y métodos}

La prueba tuvo lugar en un salón hermético que contiene cuatro nacedoras en una planta de incubación, ubicada vía San Gil-Socorro, km 9, en la recta de la vereda el Cucharo, municipio de Pinchote, departamento de Santander, georreferenciado $6^{\circ} 32^{\prime}$ latitud norte, $73^{\circ}$ 11' longitud oeste.

Se seleccionó un lote de huevos (A9) de gallinas reproductoras de la línea Cobb-avian 48, con edades de 32 y 33 semanas de vida, 15.552 huevos de la misma edad de postura fueron cargados en la máquina incubadora 4 de la marca Chick Master-genesis III. Al cumplir las 405 horas de iniciado el proceso de incubación se realizó la transferencia de los huevos a la máquina nacedora de la misma referencia, calibrada para mantener una temperatura de $98,2^{\circ} \mathrm{F}\left(36,7^{\circ} \mathrm{C}\right)$. Durante la transferencia se marcaron las bandejas que servirían posteriormente para realizar embriodiagnóstico y calcular el porcentaje de deshidratación de los pollitos. El estudio fue llevado a cabo mediante tres ensayos diferentes.

Ensayo 1: Lote huevo A9, 32 semanas de edad. Se evaluó la calidad del pollito cuando se apagó la resistencia 48 horas antes del nacimiento; en transferencia se descartó el 6,9\% del huevo cargado por infertilidad y se transfirieron a la nacedora 14.478 huevos.

Ensayo 2: Lote huevo A9, 32 semanas de edad. Se apagó la resistencia de la máquina 24 horas antes del nacimiento; se transfirieron 14.448 huevos y se descartó el 7,1\% por infertilidad.

Ensayo 3: Lote huevo A9, 33 semanas de edad. Se dejó que la máquina tuviera la resistencia encendida todo el tiempo; se transfirieron 14.550 huevos después de descartar el $6,4 \%$ por infertilidad.

En los tres ensayos se contó y se clasificó la totalidad del pollo nacido en pollo de primera, segunda y descartes, clasificación hecha según la cicatrización del ombligo.

Se realizó embriodiagnóstico teniendo en cuenta sólo las mortalidades tardías y picados no nacidos vivos o muertos.

\section{Resultados}

En la tabla 1 se muestra la calidad del pollito clasificado con base en el grado de cicatrización del ombligo. Se muestra por cada uno de los tres ensayos, el número de pollitos nacidos y su respectivo porcentaje.

Tabla 1. Calidad del pollito por cicatrización del ombligo

\begin{tabular}{|c|c|c|c|c|c|c|}
\hline & $\stackrel{\frac{\pi}{0}}{\overline{0}}$ & 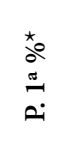 & $\begin{array}{l}\stackrel{\text { ป̃ }}{0} \\
\overline{0}\end{array}$ & 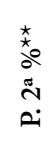 & 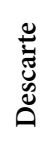 & 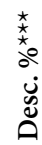 \\
\hline Ensayo 1 & 13.576 & 93,8 & 75 & 0,5 & 75 & 0,5 \\
\hline Ensayo 2 & 13.643 & 94,2 & 61 & 0,4 & 99 & 0,7 \\
\hline Ensayo 3 & 13.516 & 92,9 & 107 & 0,7 & 83 & 0,6 \\
\hline
\end{tabular}

* Porcentaje pollito de primera; ${ }^{* *}$ porcentaje de pollito de segunda; ${ }^{* * *}$ porcentaje pollito de descarte.

Fuente: elaboración propia

En el ensayo 2 se obtuvo mayor porcentaje en pollito de primera $(94,2 \%)$ en comparación con el ensayo $3(92,9 \%)$. En el ensayo 2 se obtuvo un porcentaje menor en pollitos de segunda, $0,4 \%$, en comparación con el ensayo $3,0,7 \%$.

En la tabla 2 se muestra el porcentaje de pollo deshidratado para cada uno de los tres ensayos, el total del pollito muestreado, el número de pollos con signos de deshidratación y su respectivo valor relativo.

En el ensayo 3 se obtuvo un porcentaje de pollito deshidratado de $8,81 \%$ que fue mayor en comparación a los otros dos ensayos, 3,99 en el ensayo 2 y $4,24 \%$ en el ensayo 1.

Tabla 2. Porcentaje de pollo deshidratado

\begin{tabular}{|l|c|c|c|}
\hline & $\begin{array}{c}\text { Total pollo } \\
\text { muestreado }\end{array}$ & $\begin{array}{c}\text { Pollo } \\
\text { deshidratado }\end{array}$ & $\begin{array}{c}\text { \% Pollo } \\
\text { deshidratado }\end{array}$ \\
\hline Ensayo 1 & 447 & 19 & 4,25 \\
\hline Ensayo 2 & 426 & 17 & 3,99 \\
\hline Ensayo 3 & 431 & 38 & 8,81 \\
\hline
\end{tabular}

Fuente: elaboración propia

En la tabla 3 se muestran los resultados de embriodiagnóstico y la calificación según el test físico de Cervantes. Se muestra por cada uno de los tres ensayos el porcentaje mortalidad tardía, mortalidad tardía en mala posición de los embriones, picados no nacidos y la puntuación de los pollos según el test físico de Cervantes. 
Tabla 3. Embriodiagnóstico y test físico de Cervantes

\begin{tabular}{|l|l|l|l|l|}
\hline \multicolumn{5}{|c|}{ Mortalidad embrionaria test físico de Cervantes } \\
\hline & $\begin{array}{c}\text { Mortalidad } \\
\mathbf{1 8 - 2 1} \text { días } \\
(\%)\end{array}$ & $\begin{array}{c}\text { Mortalidad } \\
\text { tardía mal- } \\
\text { posición } \\
(\%)\end{array}$ & $\begin{array}{c}\text { Picado no } \\
\text { nacido } \\
\text { vivo/muerto } \\
\%\end{array}$ & $\begin{array}{c}\text { Test físico } \\
\text { Cervantes }\end{array}$ \\
\hline Ensayo 1 & 0,8 & 0,2 & 0,6 & 94,13 \\
\hline Ensayo 2 & 0,2 & 0,0 & 0,6 & 95,30 \\
\hline Ensayo 3 & 1,0 & 0,8 & 0,6 & 90,00 \\
\hline
\end{tabular}

Fuente: elaboración propia

En el ensayo 3 la mortalidad de 18 a 21 días fue mayor con respecto a los otros dos ensayos, pues en este se obtuvo una mortalidad del $1 \%$ seguida por el ensayo 1 con un porcentaje del $0,8 \%$, y, finalmente, el ensayo 2 con la mortalidad más baja $(0,2 \%)$.

Con respecto a la malposición del embrión dentro del huevo destaca el ensayo 2 al no observarse este signo, que tuvo la mayor presencia en el ensayo 3 con el $0,8 \%$.

$\mathrm{Al}$ evaluar los huevos picados no nacidos, no se encontró diferencia entre los 3 ensayos.

En el ensayo 3 se observó en los embriones muertos en la etapa tardía de la incubación en buena o mala posición (18-21 días de desarrollo) la presencia de uratos.

El test físico de Cervantes identificó a los pollitos del ensayo 2 con la mayor calidad al obtener una puntuación de 95,30 (muy bueno), en comparación con los pollitos de los ensayos 1 y 3 con puntuaciones de 94,13 y 90,00 , respectivamente, calificando a estos como buenos.

\section{Discusión}

La revisión de la bibliografía realizada para la formulación y desarrollo de este proyecto de investigación no arrojó resultados cuando se pretendió comparar los resultados aquí obtenidos con otros estudios en los que también se apagaran las resistencias de las máquinas nacedoras horas antes de completar el ciclo de incubación. Sin embargo, sí se puede comprobar la teoría de muchos autores que aseguran que el estrés calórico afecta la calidad del pollito recién nacido.

Las nacedoras utilizadas en este estudio cuentan con un sistema de ventiladores verticales, serpentines de enfriamiento, aspersores de humedad y una resistencia de calefacción; esta última se encarga de mantener la temperatura dentro de la máquina.
La máquina está programada para mantener la temperatura a $98,2^{\circ} \mathrm{F}\left(36,7^{\circ} \mathrm{C}\right)$ y cada vez que el termómetro indique que la temperatura baja o sube de este rango, la resistencia o los sistemas de enfriamiento, según sea el caso, se encenderán para mantener la temperatura óptima dentro de la máquina [12]. Se observó en este experimento que la temperatura no es uniforme y que, por el contrario, permanece fluctuando. Apagar la resistencia cuando el mayor número de embriones ha nacido hace que ese calor embrionario producido por los pollitos sea suficiente para mantener la temperatura estable.

Se ha determinado de manera clara que cambios en la temperatura de la máquina nacedora (aumento o disminución), se convierten en un factor estresante para los pollitos y provoca un marcado efecto sobre la calidad de los recién nacidos [5]. Por otra parte, otros autores describen el efecto negativo generado por el sobrecalentamiento de los pollitos al final del ciclo de incubación, afectando el desarrollo intestinal en una magnitud similar a la que se observa cuando se priva de comida y agua a los pollos en sus primeros días de vida [13].

Como ya ha sido mencionado en este artículo, la calidad del pollito al nacimiento puede determinar la capacidad y el desempeño productivo. Al respecto se ha descrito que los pollos que fueron sometidos a altas temperaturas durante la última etapa del periodo de incubación en la máquina nacedora muestran una menor ganancia de peso, menor conversión y menor consumo hasta las tres primeras semanas [7]; sin embargo, estos datos no son desde luego directamente comparables con los obtenidos en este estudio, ya que no se realizó un seguimiento posterior a la salida de los pollitos de la planta de incubación.

Uno de los signos de mala calidad del pollito al nacimiento es la mala cicatrización del ombligo, ombligos con fugas o botones; estos datos reportados en la literatura fueron consistentes con los de esta investigación para el grupo en el cual no se apagó la resistencia de la máquina nacedora $[4,14]$.

Se ha determinado que los pollitos que presentan botones en sus ombligos, comparados con aquellos que tienen sus ombligos sanos, no presentan diferencias en la mortalidad. Sin embargo, sí tienen ventajas en la conversión y ganancia de peso, siendo mayor en los de ombligo sano [14].

En otro problema asociado al ombligo, conocido como fuga, se ha descrito que existe menor ganancia de peso respecto a los animales de ombligo sano. 
Probablemente la condición del ombligo condiciona el desarrollo y crecimiento de las aves, porque la energía que por lo regular se utilizaría para el crecimiento es desviada para cubrir problemas como infecciones o enfermedades subclínicas, generando disminución en la ganancia de peso y variabilidad en la producción de las aves [15]. Por tanto, como pudo verse en los resultados presentados arriba, el apagado de las resistencias delas máquinas nacedoras puede ofrecer ciertas ventajas.

La incubación y la calidad del pollito son objeto permanente de investigación alrededor de todo el mundo. Los investigadores siempre concluyen que el pollito de mala calidad va a tener un desarrollo ineficiente y que las máquinas de carga única son la vía para conseguir pollitos de mejor calidad. En los países en desarrollo la mayoría de las plantas de incubación cuentan con máquinas multicarga sin actualizaciones de sistemas de ventilación y sistemas de enfriamiento [5]. La respuesta puede estar en la optimización de los procesos y el trabajo con la tecnología existente, buscando la eficiencia necesaria para competir en otros mercados mientras se está a la vanguardia tecnológica de la industria avícola mundial.

\section{Conclusiones}

Los mejores resultados fueron obtenidos cuando se apagó la resistencia de la máquina nacedora 24 horas antes del nacimiento (ensayo 2). En este momento, la mayor cantidad de embriones ya había nacido; probablemente esto hizo que la temperatura se tornara más estable, disminuyendo el estrés calórico, permitiendo menor mortalidad en etapas tardías y mayor número de pollitos de primera.

Cuando la máquina funcionó normalmente (ensayo 3) se encontraron mortalidades tardías altas en relación con los ensayos en los que la resistencia de la máquina nacedora se apagó 24 y 48 horas antes del nacimiento (ensayo 2 y 1 , respectivamente), lo que indica que el cambio del funcionamiento normal de la máquina nacedora permitió obtener mayor cantidad de pollitos nacidos, en relación con el huevo transferido a esta.

En el ensayo 2, a pesar de tener mortalidad tardía muy baja y no observar el signo de mala posición de los embriones, sí se observó un incremento en los pollitos de desecho en relación con los otros dos ensayos. Pero esto se vio recompensado al obtener menos pollos de segunda y un mayor porcentaje de pollitos de primera por más de un punto por encima del ensayo, donde la máquina funcionó normalmente.

Los pollos nacidos en el ensayo en el que la máquina funcionó normalmente (ensayo 3) mostraron en un porcentaje alto algún signo de deshidratación, lo cual se reflejó en la evaluación del test físico de Cervantes, que calificó a los pollos de este ensayo como buenos; mientras que en el ensayo 2, en el que la temperatura sólo fue regulada por el calor embrionario, fue más uniforme y los embriones tenían signos de deshidratación en un porcentaje de menos de la mitad del ensayo 3, y el resultado del test físico de Cervantes fue muy bueno.

\section{Referencias}

[1] Mora SJ. La Producción Avícola. Connotaciones. Universidad Nacional de Colombia, sede Medellín. 2012. 7 p.

[2] Fenavi (Federación Nacional de Avicultores de Colombia). Programa de Estudios Económicos; Producción avícola y encasetamientos anual (1980-2010); 2011. [Consultado: 25 de junio del 2012]. Disponible en: http://www.fenavi.org/index.php?option=com_conten t\&view=article\&id=2472\&Itemid=13301

[3] Cobb Vantress. Guía de manejo de la incubadora. [Libro electrónico]. Arkansas: Cobb-Vantress Inc.; 2008. [Consultado: 29 de mayo del 2012]. Disponible en: http://www.cobb-vantress.com/contactus/brochures/ Hatchery_Guide_Spanish_2008.pdf

[4] Pachón LA. Factores determinantes de un pollito de buena calidad. Amevea: Quito, Ecuador; 2007. [Consultado: 25 de mayo del 2012]. Disponible en: http:// www.amevea-ecuador.org/datos/Factores_Determinantes_de_un_Pollito_de_Buena_Calidad.PDF

[5] Leandro NSM et al. Incubabilidade e Qualidade de Pintos de Ovos Matrizes de Frangos de Corte Submetidos a Estresse de Temperatura. Rev Bras Cienc Avic. Ene/ Abr 2000; 2(1). [Consultado: 23 de julio del 2012]. Disponible en: http://www.scielo.br/scielo.php?script=sci_ arttext\&pid=S1516-635X2000000100006\&lng=en\&nr $\mathrm{m}=$ iso.

[6] North MO, Bell, DD. Factores que afectan la incubabilidad. En: Manual de producción avícola. México: El Manual Moderno; 1998. p. 118-20.

[7] Torres A. A influência do estresse térmico no nascedouro sobre a imunidade humoral e as características zootécnicas de frangos de corte. Universidade Estadual Paulista. Faculdade de Ciências Agrárias e Veterinárias; 2006.

[8] Hamminga B, Calil T. La planta de incubación del futuro. Zedam (Países Bajos)-Rio-Claro (Brasil). s. d. 
[Consultado: 4 de septiembre del 2012]. Disponible en: http://www.amevea-ecuador.org/datos/Tomas $\% 20$ Calil\%20La\%20planta\%20de\%20incubacion \%20 del\%20futuro.pdf

[9] Meijerhof R. Influencia de la incubación en la calidad del pollito de un día. s. d. [Consultado: 14 de agosto del 2012]. Disponible en: http://www.wpsa-aeca.es/ aeca_imgs_docs/01_02_17_incubacion.pdf

[10] Boarjen M. La incubadora de carga única es la opción más natural. Selecciones Avícolas. 2005; 47(5): 287-90. [Consultado: 8 de julio del 2012]. Disponible en: http:// europa.sim.ucm.es/compludoc/AA? $\mathrm{a}=$ Boerjam $\% 2 \mathrm{c}+$ Marleen\&donde $=\& z f r=$

[11] Sandoval A et al. Aplicación de la embriodiagnosis para evaluar la eficiencia de la planta de incubación de barrilleros en una empresa avícola comercial en la Argentina. Revista de Investigación Agropecuarias. Ago 2005; 34(2): 75-80. [Consultado: 28 de mayo del 2012]. Disponible en: http://redalyc.uaemex.mx/pdf/864/86434 206.pdf
[12] Chick Master ${ }^{\circledast}$. Nacedora classic Chick Maaster ${ }^{\circledast}$. [folleto] Estados Unidos.

[13] Fribourg S. Mejora de la productividad a través de la calidad del pollito al primer día de edad. Sistema de Revisiones en Investigación Veterinaria de San Marcos. Lima: Universidad Nacional Mayor de San Marcos; 2008. [Consultado: 27 de mayo del 2012]. Disponible en: http://veterinaria.unmsm.edu.pe/files/Susana_productividad_pollito.pdf

[14] Odea E. Evaluando calidad del pollito, ¿es un ombligo sin sanar sinónimo de mal pollo parrillero? Poultry Research Centre: 2007; 3. [Consultado: 7 de junio del 2012]. Disponible en: http://www.poultryresearchcentre.com $/ \mathrm{kms} /$ files/Factsheet $\% 203 \% 20$ Chick $\% 20$ Navel\%20IRT\%20-\%20Spanish.pdf

[15] Fasenco G, O’Dea E. Evaluating broiler growth and mortality in chicks whit minor navel conditions at hatching. Poult Sci. 2007; 87(3): 594-7. [Consultado: 9 de junio del 2012]. Disponible en: http://ps.fass.org/content/87/3/594.full.pdf 